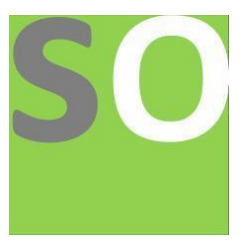

Article title: Generalized Inflation Theory as a solution to Galaxies Rotation Curves And Variation of fine structure constant

Authors: Manouchehr Amiri[1]

Affiliations: Tehran Azad University[1]

Orcid ids: 0000-0002-0884-8853[1]

Contact e-mail: manoamiri@gmail.com

License information: This work has been published open access under Creative Commons Attribution License http://creativecommons.org/licenses/by/4.0/, which permits unrestricted use, distribution, and reproduction in any medium, provided the original work is properly cited. Conditions, terms of use and publishing policy can be found at https://www.scienceopen.com/.

Preprint statement: This article is a preprint and has not been peer-reviewed, under consideration and submitted to ScienceOpen Preprints for open peer review.

DOI: 10.14293/S2199-1006.1.SOR-.PPR8FBI.v1

Preprint first posted online: 05 October 2020

Keywords: Fine structure constant variation, Galaxies rotation curve, MOND, Dark matter 


\title{
Generalized Inflation Theory as a solution to Galaxies Rotation Curves And Variation of fine structure constant
}

\author{
Manouchehr Amiri, Tehran Azad University, Iran
}

\begin{abstract}
In this paper, I propose a single model for description of both rotation curve and variation of fine structure constant at the distant stars of observable galaxies. This model generalizes the inflation of universe from spatial parameters to all phase space parameters. This achievement was made by an extension of BDM theory which was introduced by the author in previous article [1]. The generalized inflation extends the inflation concept to linear and angular momentum and interprets Galaxy rotation curve and variation of fine structure constant.
\end{abstract}

Keywords: Fine structure constant variation, Galaxies rotation curve, MOND, Dark matter.

\section{Introduction}

With a few exception, all models aimed at description of spiral galaxies rotation curves have been dismissed. The two remaining models i.e. NBDM (Non-Baryonic Dark Matter) and MOND (Modified Newtonian Dynamics) have been challenged for their unsatisfactory results or mysterious nature [1]. Velocity (rotation) curves of distant stars relative to their galaxy's center do not obey the Newtonian dynamics. Based on astronomical observations, the orbiting velocities of these stars are approximately equal or slowly increasing over the large distances whereas the Newtonian dynamic predicts a lowering speed proportional to $\frac{1}{\sqrt{r}}$ with $r$ as the distance of star from galaxy's center [2]. The problem has been justified by assuming a hidden dark matter distributed in the galaxies with a certain pattern. The modified Newtonian dynamics (MOND) is also another approach to this discrepancy by suggesting a modification of Newtonian dynamics at very large distances and very low gravity accelerations [2]. The fine structure constant variation over large astronomical distances is somehow a similar problem observed at far galaxies. I suggest an interpretation of both rotation curve of galaxies and variation of fine structure constant in the context of BDM (Binary data matrix) theory. The main idea of this article is to unify both problems in a single generalized model. By invoking the results of Binary Data Matrix, which was introduced in my previous article, the phase space parameters including spatial coordinates, linear and angular momentum are complying the universe inflation law. I call this notion as generalized inflation show that the trivial inflation of linear momentum is directly interrelated with spatial expansion whereas the inflation of angular momentum results in an asymptotic velocity for far stars of galaxies and variation of fine structure constant.

\section{Rotation (velocity) curve problem as the results of generalized inflation}

From the main equations of the BDM model that introduced in previous article [1] we conclude that all base vectors of all phase space parameters inflate through time through the equation:

$$
\langle E\rangle=\frac{1}{2} \frac{\partial}{\partial \tau} \log g
$$


where $g$ is the determinant of parametric space metric which include base vectors of all parameters i.e. spatial, linear momentum and angular momentum. This equation reveals that in BDM, the inflation involves all parameters and not only the spatial dimension and consequently it implies a generalized inflation. Obviously, the metric of bounded system by force fields does not obey the relation and therefor for system of galaxies stars, the expansion of metrics occurs at large astronomical distances where the gravity could be neglected. Due to equation (1) the rate of inflation is proportional to energy content of the system. For universe, the expansion rate is proportional to its energy or mass content and works at large distances. If $a$ stands for the scale factor of this inflation, then the ratio $\frac{\dot{a}}{a}$ equals the Hubble constant $H$ :

$$
H=\frac{\dot{a}}{a}
$$

As a generalization to inflation of spatial metric, equation (1) guarantees the inflation of all parameters with equal footing. Therefore, as the Hubble law describes the expansion rate through such an equation:

$$
H=\frac{\dot{a}}{a}=\frac{\dot{r}}{r}
$$

Due to generalized inflation principle, we could also apply the same equation for linear and angular momentum:

$$
H=\frac{\dot{p}}{p}=\frac{\dot{L}}{L}
$$

For linear momentum we obtain: $\quad \dot{p}=p H \rightarrow \dot{v}=v H \rightarrow v=r H \rightarrow r \cong r_{0} e^{H t}$

Which is compatible with Hubble law. For angular momentum we have:

$$
\dot{L}=L H \rightarrow \frac{d(v r)}{d t}=v r H \rightarrow \log v r=H t \rightarrow v r=v_{0} r_{0} e^{H t}=v_{0} r
$$

Or:

$$
v r=v_{0} r \rightarrow v=v_{0}
$$

Here $v$ stands for orbiting velocity of stars and $v_{0}$ is a constant velocity. These equations are valid at the limit of a far distance $D_{0}$ from other galaxies where the gravitational acceleration amounts to negligible limit and the observable inflation begins. At this critical distance which is about 2-4 Mpc (Mega Parsec) the radial velocity respect to Hubble's law start to be observable and yields:

$$
v_{0}=D_{0} H
$$

The initial velocity $v_{0}$ should be interpreted as the escape velocity which determines the lower limit of expansion velocity. Consequently, the orbit velocity $v$ also equals this escape velocity and obeys the expansion effects in the negligible gravity. Then we have:

$$
v=v_{0}=D_{0} H
$$

Evidently the observable expansion of galaxies is possible for galaxy's stars at very large distances where the gravity loses required binding potential to hinder expansion of spatial and non-spatial parameters. Based on astronomical observations, the least distance $D_{0}$ is about 2-4 Mpc (Mega Parsec). Then the estimated constant velocity $v_{0}$ will be read as (with $H \cong 70 \mathrm{~km} / \mathrm{s}$. Mparsec :

$$
v_{0} \cong D_{0} H=140-280 \mathrm{~km} / \mathrm{s}
$$


Surprisingly, this is the range of asymptotic constant velocities of the stars at the rim of galaxies. The acceleration emerged from generalized inflation at large distances will read as:

$$
a=\frac{v_{0}^{2}}{r}=\frac{\left(D_{0} H\right)^{2}}{r}
$$

This acceleration replaces the Newtonian acceleration around the distance $D_{0}$ and dominates it at the astronomical distances comparable to it. Therefore, we get two separable acceleration, Newtonian which

is proportional to $\frac{1}{r^{2}}$ and BDM derived acceleration that is proportional to $\frac{1}{r}$. At the astronomical distances the Newtonian acceleration decreases rapidly and $v$ approaches to $D_{0} H$ as the final constant velocity that corresponds to $v_{\infty}$ in MOND theory. Equation that fits almost all observed data of galaxies rotation curves could be extrapolated in a way followed by MOND approach to connect these limits of velocities at the far and near astronomical distances. At this level it is compatible with MOND results after clarifying the reason for Newtonian modification at very large distances based on BDM theory results.

This reveals the reason for rotation curve discrepancies without invoking the existence of hidden Dark matter or phenomenological modification of Newtonian dynamics (MOND). For far orbiting stars in a galaxy, the angular momentum obeys the Newtonian dynamics up to a distance where the expansion of parameters overcomes the gravitational field acceleration. Of course, this velocity is equal to asymptotic velocity of stars in the galaxy disk as assumed in the context of MOND theory and be achieved by stars when the distances are about $D_{0}=2-4 \mathrm{Mpc}$ from galaxy center. Stars speed behave asymptotically between the points where we arrive the fundamental acceleration $a_{0}$ and the point of critical distance $D_{0}$ which have been observed through astronomical data. Here we proved the relation between the Hubble constant, orbit velocity limit and $D_{0}$ as the minimum distance where the inflation begins.

\section{Physical constants and generalized inflation}

The spin of particles as an internal angular momentum with its involvement in electromagnetic interaction between electron and photon, is included in the parametric space. The any generalized inflation that evolves the angular momentum as described in previous section, would impact on electron spin. The electron spin $S$ with the value of $\frac{\hbar}{2}$ will change over time due to the equation (5):

$$
S=S_{0} e^{H t}
$$

If we calculate the relative change of spin over 1 year, we get:

$$
\frac{\dot{s}}{S} \cong 6 \times 10^{-11} / \text { year }
$$

Fine structure constant $(\alpha)$ is among the most suspicious constant which should be evolved over universe evolution. The definition for this electromagnetic coupling constant is:

$$
\alpha=\frac{e^{2}}{\hbar c}
$$

$e$ and $c$ are constants that are not included in parameters of BDM and therefor are not involved by inflation evolution. However, $\hbar$ as an angular momentum will alter over inflation and results in negative relative change to $\alpha$. Consequently, the rate of relative change of $\alpha$ reads as: 


$$
\frac{\dot{\alpha}}{\alpha}=\frac{\dot{S}}{S} \cong 6 \times 10^{-11} / \text { year }
$$

This change rate is compatible with one of the related data was given by Shylakhter [2] and reviewed by Lipovka and Cardenas $[3,4]$ which is reported as $4 \times 10^{-11}$. This shows an amazing compatibility of BDM generalized inflation results and astronomical results of physical constants evolution.

\section{Conclusion}

By introducing the concept of generalized inflation (expansion), I extend the cosmic inflation to a broader concept including all phase space physical parameters. This generalization was achieved by the results of Binary Data Matrix model [1]. Incorporating the linear and angular momentum with spatial parameters in universe expansion leads to a formalism that interprets both rotation curve of galaxies and evolution of fine structure constant. Therefor this model may be considered as a potent alternative for NBDM and MOND theories. Further works along with this notion, may result in the solutions to solar system angular momentum problem and spiral structure of galaxies.

\section{References}

1) Amiri, M. On Equivalence of Quantum Liouville Equation and Metric Compatibility Condition, a Ricci Flow Approach. Preprints 2016, 2016120049 (doi: 10.20944/preprints201612.0049.v1).

2) Hofmeister, Anne M., and Robert E. Criss. "Debated Models for Galactic Rotation Curves: A Review and Mathematical Assessment." Galaxies 8.2 (2020): 47.

3) Troitskii, V. S. "Physical constants and evolution of the universe." Astrophysics and Space Science 139.2 (1987): 389-411.

4) Lipovka, Anton, and Iván A. Cárdenas. "Variation of the fine structure constant." (2016). 